\title{
Subplcosecond Time-Resolved Intramolecular Electronic Energy Transfer in Flexible Bichromophoric Coumarin Molecules
}

\author{
M. Kaschke, ${ }^{* \dagger}$ N. P. Ernsting, ${ }^{*}$ \\ Max-Planck-Institut für biophysikalische Chemie, Abteilung Laserphysik, D-3400 Göttingen, \\ Federal Republic of Germany \\ B. Valeur,* and J. Bourson* \\ Laboratoire de Chimie Gënèrale, (CNRS URA 1103) Conservatoire National des Arts et Mêtiers, \\ 292 rue Saint-Martin, 75003 Paris, France (Received: November 21, 1989; In Final Form: February 23, 1990)
}

By excite-and-probe spectroscopy with subpicosecond time resolution, we have measured the intramolecular electronic energy transfer in bichromophoric coumarins linked by a flexible polymethylene chain. The transfer proceeds on a time scale between 1 and 20 ps depending on the polymethylene chain length. The results can be well described by a dipole-dipole interaction model that takes into account the statistical distribution of intramolecular distances between the two chromophores.

\section{Introduction}

Intramolecular radiationless electronic-energy-transfer processes in bichromophoric molecules form a subject of considerable interest because of their implications in numerous fields: photophysics, photochemistry, biology, laser physics, and polymers. ${ }^{1}$

In particular, in dye laser physics bichromophores may be used to increase the laser efficiency by combining a higher absorption cross section (due to the donor chromophore) for the pump wavelength $\left(\mathrm{XeCl}^{*}\right.$ laser, $\mathrm{N}_{2}$ laser) with a high fluorescence quantum yield (due to the acceptor chromophore) in the visible spectral range. ${ }^{2}$ The great utility of this concept for flashlamp-pumped dye lasers has already been demonstrated. ${ }^{2,3}$

For separations between donor and acceptor that are larger than a few angstroms, the dominant mechanism for energy transfer will be the Förster dipole-dipole interaction. ${ }^{4}$ The rate constant of transfer in this case is given by

$$
k_{\mathrm{ET}}=\frac{9000[\ln (10)] \phi_{\mathrm{D}} \chi^{2}}{128 \pi^{5} n^{4} N_{\mathrm{L}} \tau_{\mathrm{D}}} \frac{S}{R^{6}}=\frac{3}{2} \chi^{2} \frac{1}{\tau_{\mathrm{D}}} \frac{R_{0}{ }^{6}}{R^{6}}
$$

$\phi_{D}$ is the donor fluorescence quantum yield, $\tau_{D}$ the donor lifetime in the absence of the acceptor, $N_{\mathrm{L}}$ Avogadro's number, $n$ the refractive index, $\chi^{2}$ an orientation factor arising from the relative orientation of donor and acceptor transition dipoles, and $R$ their mutual distance. $R_{0}$ is the so-called Förster radius. ${ }^{4} S$ represents the spectral overlap of the normalized donor fluorescence quantum distribution over wavenumbers per centimeter, $f(\tilde{v})$, with the acceptor extinction coefficient $\epsilon$ in $\mathrm{L} \cdot \mathrm{mol}^{-1} \cdot \mathrm{cm}^{-1}$ :

$$
S=\int f(\tilde{\nu}) \epsilon(\tilde{\nu}) \tilde{\nu}^{-4} \mathrm{~d} \tilde{\nu}
$$

with

$$
\int f(\tilde{\nu}) \mathrm{d} \tilde{\nu}=1
$$

The linkage of the two chromophores can be either rigid ${ }^{5}$ or flexible. ${ }^{6.7}$ In the first case, excitation energy transfer is determined by a single rate constant, whereas in the latter case a distribution of distances $R$ and of orientation factors $\chi^{2}$ has to be taken into account.

Information on the dynamics of intramolecular energy transfer can be obtained by means of up-conversion of the acceptor fluorescence, ${ }^{8}$ phase modulation ${ }^{9}$ techniques at donor and acceptor fluorescence wavelengths, or ground-state-recovery measurements of the donor chromophore. ${ }^{7}$ In principle, the distributions of interchromophoric distances can be extracted from energy-transfer experiments by analyzing the temporal evolution of the excited-

\footnotetext{
On leave from the Akademic der Wissenschaften der DDR, Zentralinstitut für Optik und Spektroskopie, DDR 1199, Berlin.
}

state population of the donor or of the acceptor chromophore. Generally, subpicosecond time resolution is required to fully resolve the transfer process between closely linked laser dye molecules. It should be mentioned that steady-state measurements of transfer efficiencies by various methods ${ }^{10}$ are inadequate for gaining information on the distribution of distances and orientations in this case.

In this paper we present subpicosecond time-resolved exciteand-probe measurements of electronic energy transfer in flexible bichromophoric coumarin dyes after excitation with a short UV pulse at $308 \mathrm{~nm}$, and covering the spectral probe range of $380-675$ $\mathrm{nm}$. The linkage in these bichromophores is a polymethylene chain $-\left(\mathrm{CH}_{2}\right)_{n}$ - with $n=3,4,8$, and 12 (see Figure 1). As model compounds 7-ethoxycoumarin and coumarin 500 are used for the donor chromophore $\mathrm{D}$ and acceptor chromophore $\mathrm{A}$, respectively. Figure 2 shows their cross sections for absorption and for stimulated emission.

The energy-transfer process within the bichromophoric compound (labeled DnA) is studied by measuring the temporal evolution of the gain in the spectral region of the acceptor chromophore fluorescence. The aim of our work is to get direct experimental proof for the recently calculated ${ }^{11}$ statistical distribution of distances due to the various possible conformations of such a flexible polymethylene chain.

\section{Experimental Section}

The synthesis of the bichromophores has been described in ref 6. For the subpicosecond spectroscopic measurements, samples were prepared in methanol (Merck UVASOL). They were contained in $\mathrm{CaF}_{2}$ flow cells with a path length of $1 \mathrm{~mm}$. Small-signal optical densities at $308 \mathrm{~nm}$ were adjusted to values

(1) (a) De Schryver, F. C.; Boens, N.; Put, J. Adv. Photochem. 1977, 10 359. (b) Valeur, B. In Fluorescent Biomolecules; Jameson, D. M., Reinhardt, G. D., Eds.; Plenum Press: New York, 1989; p 296.

(2) Schäfer, F. P.; Bor, Zs.; Lüttke, W.; Liphardt, B. Chem. Phys. Lett. $1978,56,454$.

(3) Huth, V. Dissertation, Siegen, 1988.

(4) Förster, Th. Z. Naturforsch. 1949, 4a, 321. 61.

(6) Bourson, J.; Mugnier, J.; Valeur, B. Chem. Phys. Lett. 1982, 92, 430 (7) Ernsting, N. P.; Kaschke, M.; Kleinschmidt, J,; Drexhage, K. H.; Huth, V. Chem. Phys. 1988, l22, 431

(8) Kopainsky, B.; Kaiser, W.; Schäfer, F. P. Chem. Phys. Lett. 1978, 56, 458

(9) Mugnier, J.; Valeur, B.; Gratton, E. Chem. Phys. Lett. 1985, 119, 217 (10) Mugnier, J.; Pouget, J.; Bourson, J.; Valeur, B. J. Lumin. 1985, 33 , 273

(11) Valeur, B.; Mugnier, J.; Pouget, J.; Bourson, J.; Santi, F. J. Phys. Chem. 1989, 93, 6073 . 


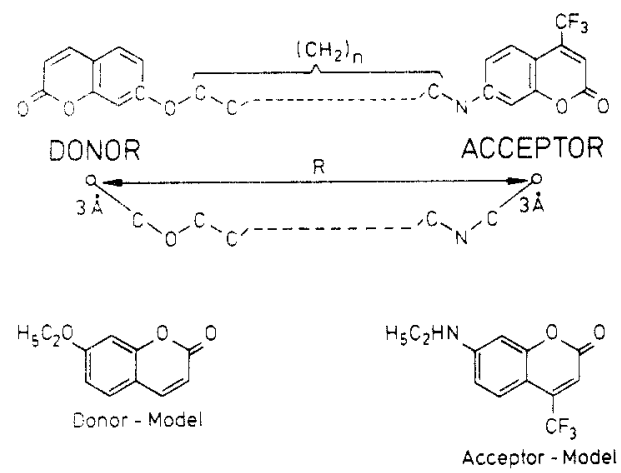

Figure 1. Molecular structure of bichromophoric coumarins and model compounds of donor and acceptor.

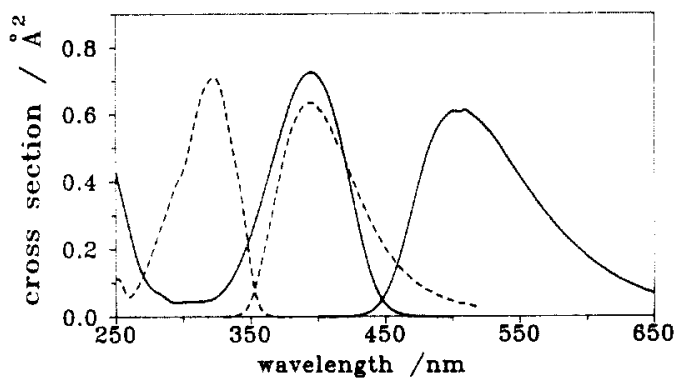

Figure 2. Cross sections for absorption and stimulated emission of the model donor chromophore (dashed lines) and of the model acceptor chromophore (solid lines).

around 0.5 to obtain almost homogeneous excitation along the cell path.

Absorption spectra were recorded on a Shimadzu spectrophotometer with a spectral resolution of $2 \mathrm{~nm}$. Fluorescence spectra were measured on a Spex 212 fluorometer, with a bandwidth of $1 \mathrm{~nm}$ for emission, and using a depolarizer in front of the emission monochromator. In order to avoid reabsorption of fluorescence, front surface collection was chosen, and the optical density of the sample was less than 0.05 .

The laser system combined a colliding-pulse-mode dye laser with a $\mathrm{XeCl}^{*}$ excimer laser (for details see ref 12 ). It produces intense pump pulses at $308 \mathrm{~nm}$, with pulse duration of $0.26 \mathrm{ps}$ ( $\mathrm{fwhm}$ ), and pulses at $616 \mathrm{~nm}$ of 0.24 -ps duration. The red pulses are used for the generation of a spectral continuum that probes the transient absorption of the excited sample. The temporal evolution of the transient spectra was followed by varying the delay between the pump and probe pulses.

\section{Results}

A typical sequence of transient spectra for various delay times is given in Figure 3 for the bichromophore D8A. We have measured the change in optical density in the wavelength range $380-675 \mathrm{~nm}$. The polarization of the probe pulse in this case was at the magic angle with respect to that of the pump pulse. In the range $400-440 \mathrm{~nm}$, we observe an increase in absorption due to an excited state $\rightarrow$ excited state transition in acceptors that have been excited by energy transfer via the linked donor chromophores. The assignment of this absorption band to the excited acceptor state was confirmed by an independent pump/probe measurement with the acceptor model molecule alone. In the range 460-575 $\mathrm{nm}$, we observe gain due to stimulated emission from excited acceptor molecules.

Figure 4 shows kinetic curves (gain rise) derived from the transient absorption spectra at the probe wavelength of $500 \mathrm{~nm}$, for the bichromophores D3A, D4A, D8A, and D12A. For comparison, the relative change of optical density for a solution of DASBTI ([2-[ $p$-(dimethylamino)styryl] benzothiazolyl]ethyl iodide) at $500 \mathrm{~nm}$ is also shown. This change is due to the removal of DASBTI from its electronic ground state by optical pumping;

(12) Ernsting, N. P.; Kaschke, M., to be published

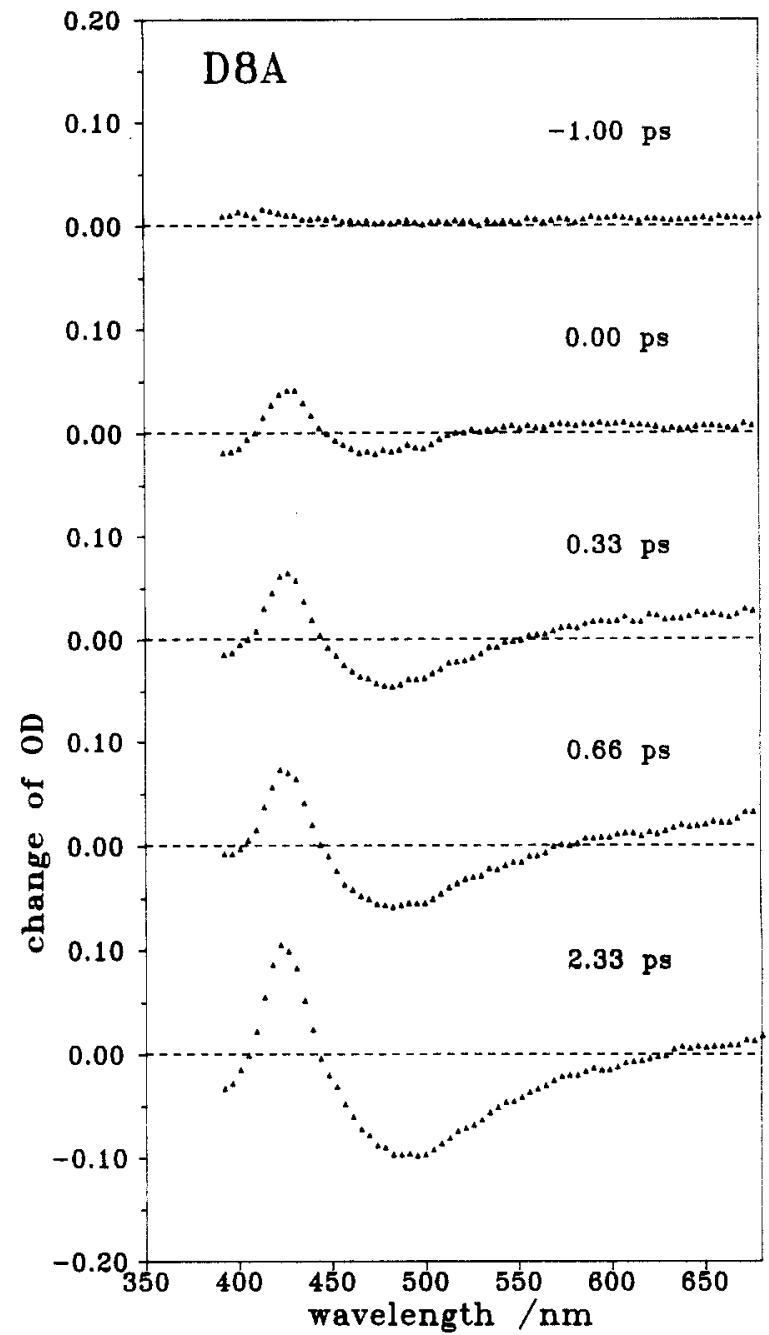

Figure 3. Transient spectra of changes in optical density of the bichromophoric coumarin D8A for various delay times after excitation with a $0.3-p s$ UV pulse. The spectra shown represent an accumulation of 100 laser shots. No smoothing or other data treatment procedure has been applied.

therefore it gives a measure of our time resolution. Let us return to the bichromophoric coumarins. For each of these compounds, the characteristic transient absorption at $425 \mathrm{~nm}$ shows exactly the same temporal evolution as the gain dynamics. This confirms our assignment of the transient absorption band to excited-state absorption by the acceptor molecule. Comparing parts a-c of Figure 4, the kinetics of energy transfer are clearly distinguished: the process becomes slower with increasing chain length.

\section{Discussion}

4.1. Distribution of Distances. In a recent paper by two of the authors, ${ }^{11}$ the distribution of donor-acceptor distances in flexible bichromophoric molecules has been calculated. This was done by using rotational isomer theory together with the statistical weights for the conformations of short sequences of three and four bonds; it is thus possible to calculate the probability of a given conformation of the whole molecule. For each conformation, the center-to-center distance can easily be obtained from geometric considerations taking into account the valence angles, the internal rotation angles, and the size of the chromophores. In this way one obtains the distribution of distances shown in Figure 5 for the four bichromophoric coumarin molecules that are studied here experimentally. For the bichromophores D3A and D4A, the probability for each of the three or four bond configurations (each corresponding to a certain intramolecular distance $R$ ) has been plotted. Owing to the large number of possible configurations for the bichromophores D8A (3362) and D12A (114243), the probabilities are averaged over intramolecular distance with a step 
Electronic Energy Transfer in Coumarin Molecules
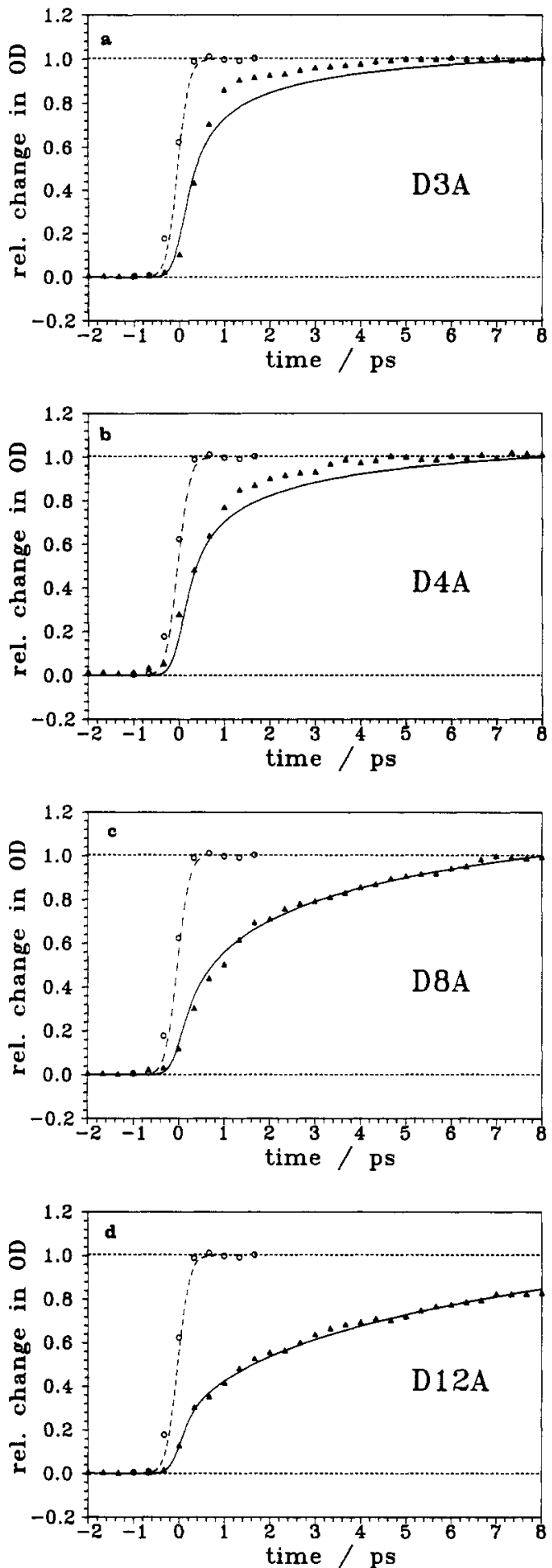

Figure 4. Experimental rise of acceptor gain at $500 \mathrm{~nm}$ (negative changes of optical density) for various bichromophoric coumarins DnA after excitation with a 0.3 -ps UV pulse (triangles). For comparison the "instantaneous" bleaching of DASBTI at $500 \mathrm{~nm}$ is indicated by the circles. The results of model calculations are given by the solid line. Again for comparison, the "instantaneous" bleaching of DASBTI has been calculated (dashed line) assuming a 0.3 -ps probe pulse.

size of $0.5 \AA$. Therefore, the probability densities are shown in these cases. A detailed description of the calculation procedure can be found in ref 11 .

4.2. Distribution of Relative Orientations. In addition to having a distribution of distances $R$, the linked donor and acceptor chromophores also attain a range of relative orientations. A particular relative orientation may be described by only two angles $\Phi$ and $\theta,{ }^{13}$ and the orientation factor in (1) becomes

$$
\chi^{2}(\Phi, \theta)=\left(3 \cos ^{2} \Phi+1\right) \cos ^{2} \theta
$$
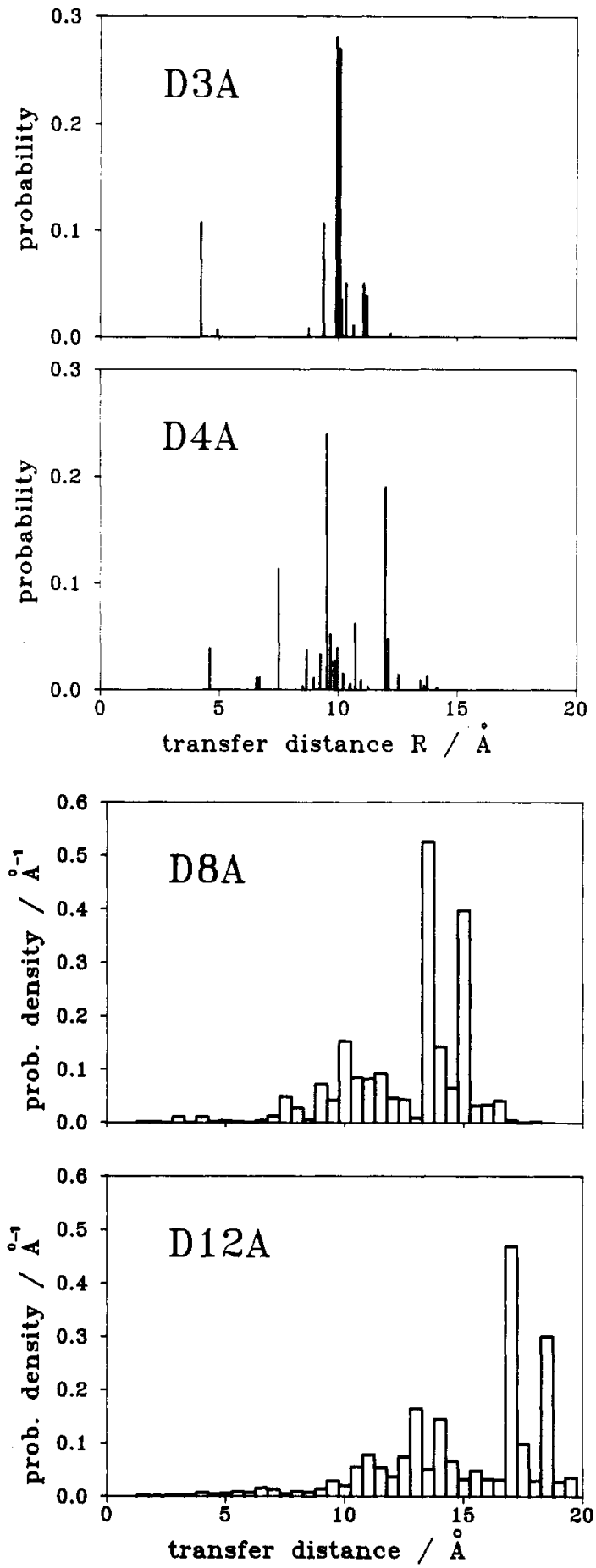

Figure 5. Distribution of intramolecular distances for bichromophores D3A, D4A, D8A, and D12A as calculated in ref 11 .

where $0 \leq \Phi, \theta \leq \pi$.

The question now is whether there is a preferred orientation of the acceptor with respect to the donor, especially for small intramolecular separations between donor and acceptor chromophores, or if the chromophores are randomly oriented. [It should be noted that this orientational distribution (random or correlated) can be assumed to be static on the time scale of the transfer process (a few picoseconds).] Emission anisotropy measurements (or equivalently measurements of gain anisotropy due to acceptor-excited-state stimulated emission) can bring answers to this question. From steady-state-fluorescence depolarization ${ }^{10}$ it can be concluded that nonrandomicity of mutual orientation can be only very small if any. This random orienta-

(13) Steinberg, I. Z. J. Chem. Phys. 1968, 48, 2411 

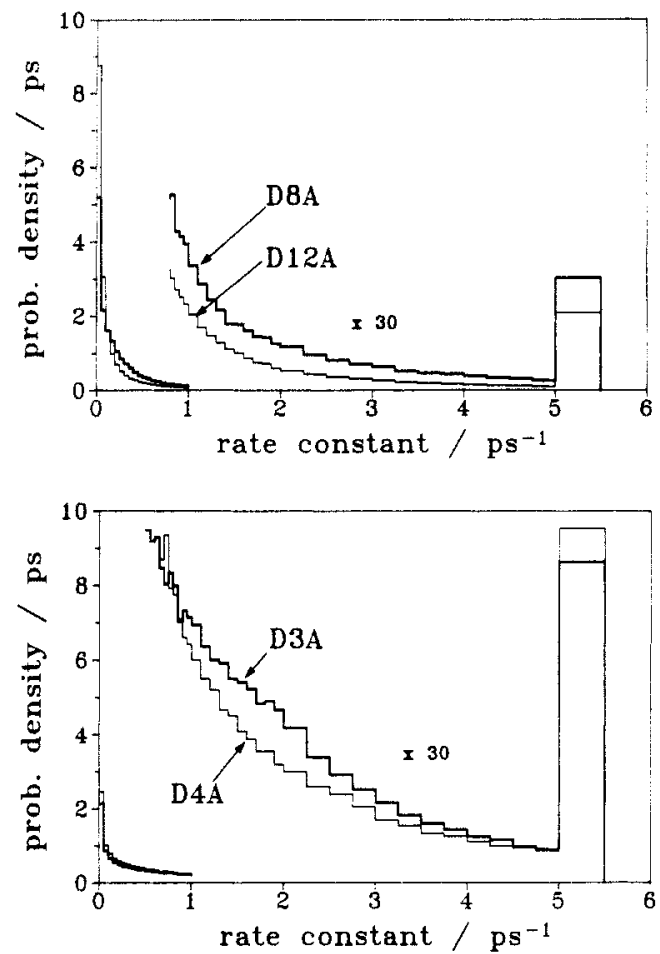

Figure 6. Distribution of intramolecular energy transfer rate constants, calculated from the distribution of distances and assuming random relative orientation of the two linked chromophores.

tional distribution can be understood if one considers that the chromophores are allowed to rotate around the axis of the link with the chain, the axis being not in the same direction as the transition dipole. Moreover, there are several conformations corresponding to almost identical end-to-end distances but with different mutual orientations of donor and acceptor. Further evidence has been provided recently by Ikeda et al. ${ }^{14}$ who showed that in $\alpha, \omega$-bis(naphthyl)alkanes with 3,6,7, and 12 methylene groups, the orientational distribution of the two chromophores is so broad that the system may be considered as randomly oriented. Therefore, we assume for our model random distribution of angles $\Phi$ and $\theta$, independent of each other and also independent of the distance $R$ between the chromophoric centers.

4.3. Distribution of Energy-Transfer Rate Constants. For each of the bichromophoric compounds under investigation, a distribution of energy-transfer rate constants may then be generated by use of (1) and (3). The probability densities for the rate constants are shown as histograms in Figure 6. The limiting block in the histograms requires some comment. The time resolution of our spectrometer is determined by the width of the intensity cross-correlation between the pump pulse and the probe continuum pulse. The experimental width of ca. $0.35 \mathrm{ps}$ for this cross-correlation implies that we cannot resolve processes that have rate constants larger than $5 \mathrm{ps}^{-1}$ from a process that has instantaneous response. In order to show the calculated relative contribution of unresolvably fast energy transfer to the overall kinetics, we have therefore condensed rate constants $k_{\mathrm{ET}} \geq 5 \mathrm{ps}^{-1}$ into a representative limiting interval $5 \mathrm{ps}^{-1} \leq k_{\mathrm{ET}} \leq 5.5 \mathrm{ps}^{-1}$. Accordingly this interval of rate constants has high probability density as indicated by the limiting blocks in the figure.

4.4. Model Calculations. The kinetic model employed for the simulation of intramolecular energy transfer in flexible bichromophores is based on a rate equation description, since electronic dephasing in solution at room temperature is expected to elude our time resolution. Further, only dipole-dipole energy transfer is taken into account. This is justified by an inspection of Figure 5 , where only a small fraction of molecular conformations has

(14) Ikeda, T.; Lee, B.; Kurihara, S.; Tazuke, S.; Ito, S.; Yamanoto, M. J. Am. Chem. Soc. 1988, II0, 8299.

(15) Baumann. J.; Fayer, M. D. J. Chem. Phys. 1986, 85, 4087
TABLE I

\begin{tabular}{|c|c|c|}
\hline \multicolumn{3}{|c|}{ Donor Molecule (7-Ethoxycoumarin) } \\
\hline$\sigma_{\mathrm{D}}^{\mathrm{a}}$ & $\begin{array}{l}\text { ground-state absorption cross section } \\
\qquad(308 \mathrm{~nm})^{a}\end{array}$ & $0.537 \AA^{2}$ \\
\hline$\sigma_{\mathrm{D}}^{\mathrm{e}}$ & donor emission cross section $(500 \mathrm{~nm})^{a}$ & $0.034 \AA^{2}$ \\
\hline$\tau_{D}$ & donor fluorescence lifetime ${ }^{b}$ (methanol) & $0.420 \mathrm{~ns}$ \\
\hline$\tau_{\text {Rad }}$ & donor radiative lifetime (methanol) $^{c}$ & $4.235 \mathrm{~ns}$ \\
\hline$\phi_{\mathrm{D}}$ & $\begin{array}{l}\text { donor fluorescence quantum yield } \\
\qquad\left(=\tau_{D} / \tau_{R a d}\right)\end{array}$ & 0.099 \\
\hline \multicolumn{3}{|c|}{ Acceptor Molecule (Coumarin 500) } \\
\hline$\sigma_{A}^{a}$ & $\begin{array}{l}\text { ground-state absorption cross section } \\
\qquad(308 \mathrm{~nm})^{a}\end{array}$ & $0.045 \AA^{2}$ \\
\hline$\sigma_{A}^{e}$ & $\begin{array}{l}\text { acceptor emission cross section ( } 500 \\
\mathrm{~nm})^{a}\end{array}$ & $0.602 \AA^{2}$ \\
\hline \multicolumn{3}{|c|}{ Spectral Overlap } \\
\hline $\begin{array}{l}S \\
R_{0}\end{array}$ & spectral overlap integral ${ }^{d}$ & $3.33 \cdot 10^{-14} \mathrm{l} \cdot \mathrm{mol}^{-1} \cdot \mathrm{cm}^{3}$ \\
\hline \multicolumn{3}{|c|}{ Pump/Probe Setup } \\
\hline$N_{\text {tot }}$ & $\begin{array}{l}\text { pump fluence } \\
\text { total number density }\end{array}$ & $\begin{array}{l}(0.5-1.0) \cdot 10^{28} \mathrm{~cm}^{-2} \mathrm{~s}^{-1} \\
2.1 \cdot 10^{17} \mathrm{~cm}^{-3}\end{array}$ \\
\hline
\end{tabular}

a Absorption spectra were recorded on a Perkin-Elmer Model 340 spectrophotometer; fluorescence spectra were measured on a Spex 212. ${ }^{b}$ From phase fluorometry. "Calculated from spectroscopic data using the Strickler-Berg formula. ${ }^{d}$ Calculated from spectroscopic data. 'Calculated with $\chi^{2}=2 / 3$ and $\phi_{D}=0.099$ (see text).

intramolecular donor-acceptor separations of less than $5 \AA$, which would make transfer by an exchange mechanism probable.

Contributions from intermolecular electronic energy transfer can be ruled out for the following reason. Given a small-signal absorbance of 0.5 in a $1-\mathrm{mm}$ cuvette and a small-signal cross section of $0.537 \AA^{2}$ at $308 \mathrm{~nm}$, we have a bichromophoric density of about $2 \times 10^{17} \mathrm{~cm}^{-3}$ (equivalent to $3.3 \times 10^{-4} \mathrm{~mol} / \mathrm{L}$ ). This corresponds to an average intermolecular separation between bichromophoric molecules of $160 \AA$, which is more than 8 times larger than the largest intramolecular distance. Since the distance enters with the sixth power in the transfer rate (1), intermolecular transfer need not be taken into account.

Higher excited electronic states of either donor or acceptor chromophore are not considered in our calculation. Their internal conversion relaxation time is assumed to be in the order of 100 $\mathrm{fs}$, which-given our pump/probe cross-correlation time of ca. $0.4 \mathrm{ps}$-allows us to neglect the transient population of these states even at earliest times. We further limit our description to bichromophores in which only the donor or only the acceptor chromophore is electronically excited. These states of the bichromophore are simply denoted $\mathrm{D}^{*}$ and $\mathrm{A}^{*}$, respectively.

The geometrical part of our model gives a probability $P_{0^{-}}$. $\left(k_{\mathrm{ET}}\right)$-based on a distribution of intramolecular distances between chromophores - that the bichromophoric molecule has a conformation which results in the rate constant $k_{\mathrm{ET}}$. In addition, let $P_{\mathrm{D}}^{*}\left(t, k_{\mathrm{ET}}\right)$ denote the probability that the molecule is donor-excited and has rate constant $k_{\mathrm{ET}}$ (and analogously for $P_{\mathrm{A}}^{*}\left(t, k_{\mathrm{ET}}\right)$ ). The absorption cross sections for pump light are denoted by $\sigma_{\mathrm{D}}{ }^{\mathrm{a}}$ and $\sigma_{\mathrm{A}}{ }^{\mathrm{a}}$ for the donor and acceptor chromophore, respectively. Then the rate equations for the probabilities read

$$
\begin{aligned}
& \mathrm{d} / \mathrm{d} t P_{\mathrm{D}}{ }^{*}\left(t, k_{\mathrm{ET}}\right)= \\
& \sigma_{\mathrm{D}}{ }^{\mathrm{a}} I_{\mathrm{p}}(t)\left[P_{0}\left(k_{\mathrm{ET}}\right)-P_{\mathrm{D}}{ }^{*}\left(t, k_{\mathrm{ET}}\right)-P_{\mathrm{A}}^{*}\left(t, k_{\mathrm{ET}}\right)\right]-k_{\mathrm{ET}} P_{\mathrm{D}}{ }^{*}\left(t, k_{\mathrm{ET}}\right)
\end{aligned}
$$

$$
\begin{aligned}
& \mathrm{d} / \mathrm{d} t P_{\mathrm{A}}^{*}\left(t, k_{\mathrm{ET}}\right)= \\
& \sigma_{\mathrm{A}}{ }^{\mathrm{a}} I_{\mathrm{P}}(t)\left[P_{0}\left(k_{\mathrm{ET}}\right)-P_{\mathrm{D}}{ }^{*}\left(t, k_{\mathrm{ET}}\right)-P_{\mathrm{A}}{ }^{*}\left(t, k_{\mathrm{ET}}\right)\right]+k_{\mathrm{ET}} P_{\mathrm{D}}{ }^{*}\left(t, k_{\mathrm{ET}}\right)
\end{aligned}
$$

The photon flux of the pump light, $I(t)$, was assumed to have a hyperbolic secant shape ( $\mathrm{fwhm}=0.3 \mathrm{ps}$ ) normalized to the pump pulse fluence. The differential equations were now solved numerically for every rate constant $k_{\mathrm{ET}}$ supporting the distributions shown in Figure 6 . The total probabilities $P_{D}^{*}(t)$ of donor excitation and $P_{\mathrm{A}}^{*}(t)$ of acceptor excitation were obtained:

$$
P_{\mathrm{D}}{ }^{*}(t)=\sum P_{\mathrm{D}}^{*}\left(t, k_{\mathrm{ET}}\right) \text { and } P_{\mathrm{A}}^{*}(t)=\sum P_{\mathrm{A}}^{*}\left(t, k_{\mathrm{ET}}\right)
$$


The kinetic transmission curve for $\lambda=500 \mathrm{~nm}$

$$
T_{500 \mathrm{~nm}}(t)=\exp \left\{d \sigma_{\mathrm{D}}{ }^{e} N_{0} P_{\mathrm{D}}{ }^{*}(t)+d \sigma_{\mathrm{A}}{ }^{e} N_{0} P_{\mathrm{A}}{ }^{*}(t)\right\}
$$

( $N_{0}$ is the number density of the bichromophoric compound, $d$ is the optical path length, $\sigma_{D}{ }^{e}$ and $\sigma_{A}{ }^{e}$ are the emission cross section at $500 \mathrm{~nm}$ of donor and acceptor chromophore, respectively) was convoluted at various delay times with a normalized Gaussian function ( $\mathrm{fwhm}=0.3 \mathrm{ps}$ ) representing the probe pulse. All parameters that were used in the calculations are collected in Table I. The resulting calculated changes in optical density, $\triangle O D=$ $-\log \left(T_{500 \mathrm{~nm}}(t)\right)$, were normalized to the maximum observed changes. The results are shown as solid lines in Figure 4 in comparison with the experimental data.

Very good agreement has been obtained for the bichromophores D8A and D12A. It should be emphasized that the simulations use parameters which have been determined by independent measurements, such as cross sections for absorption and emission, spectral overlap integral, and pump fluence. Thus we may conclude that the distribution of intramolecular radii as calculated in ref 11 , which enters significantly in our model calculations, is very well confirmed experimentally by our time-resolved measurements.

However, the simulations for D3A and D4A show systematic deviation from the measured data at delay times in the 1-7-ps range. Here the contribution of unresolvably fast intramolecular energy transfer is underestimated by our model. We believe that this deviation is most likely due to the simplifications made in the geometrical part of the model. For bichromophores linked by only three or four methylene groups, the number of molecular conformations is relatively small. Only a few-and specific-relative orientations are associated with a particular distance for energy transfer. Therefore, the assumption that the relative orientation is random and independent of distance is not confirmed by experiment in this case, as is borne out in Figure 4. Emission anisotropy measurements with high temporal resolution will reveal more detailed information on these deviations from a random orientational distribution. This will be a subject of a forthcoming paper. ${ }^{16}$

These experiments are an instructive example of how detailed information on molecular structures and their distributions can be gained from subpicosecond time-resolved measurements. To the best of our knowledge, these measurements constitute the first subpicosecond dynamic study of intramolecular energy transfer with the underlying effect of distance distribution and clear experimental distinction between different chain lengths.

Acknowledgment. We thank Profs. F. P. Schäfer and B. Wilhelmi for constant support and a critical reading of the manuscript. We gratefully acknowledge the Deutsche Forschungsgemeinschaft for support through the Leibniz Prize Program. M.K. is grateful for a Max-Planck scholarship. We also thank Dr. G. Marriott for the measurement of the donor fluorescence lifetime.

(16) Kaschke, M.; Ernsting, N. P.; Valeur, B.; Bourson, J. Manuscript in preparation.

\title{
Determination of the Electronic Structure of Thiophene Oligomers and Extrapolation to Polythiophene
}

\author{
Derek Jones, ${ }^{*}$ Maurizio Guerra, Laura Favaretto, \\ ICoCEA, Consiglio Nazionale delle Ricerche, via della Chimica, 8, 40064 Ozzano Emilia, Bologna, Italy
}

\author{
Alberto Modelli, \\ Dipartimento di Chimica “G. Ciamician”, Università di Bologna, via Selmi, 2, 40127 Bologna, Italy
}

Monica Fabrizio,

IPELP, Consiglio Nazionale delle Ricerche, Corso Stati Uniti, 4, 35020 Camin, Padova, Italy

and Giuseppe Distefano

Dipartimento di Chimica, Università di Ferrara, via Borsari, 46, 44100 Ferrara, Italy

(Received: November 27, 1989; In Final Form: February 16, 1990)

\begin{abstract}
Ionization energies, attachment energies, and electrochemical reduction potentials of thiophene oligomers $(n \leq 5)$ have been determined experimentally (ultraviolet photoelectron and electron transmission spectroscopies and cyclic voltammetry) and theoretically (ionization and attachment energies by MINDO/3). The geometrical parameters of the most stable conformation of 2,2'-bithienyl have been computed at the ab initio STO-3G level with complete relaxation. A short extrapolation of the energy data to the polymer provided accurate and reliable values for important properties of (gas phase) polythiophene, namely, ionization energy $(6.9 \mathrm{eV})$, valence bandwidth $(3.2 \mathrm{eV})$, electron affinity $(0.9-1.1 \mathrm{eV})$, HOMO-LUMO energy gap (5.9 $\mathrm{eV})$, and $\lambda_{\max }(2.7 \mathrm{eV})$.
\end{abstract}

\section{Introduction}

There has been a great deal of interest in polymeric organic compounds showing unusual electronic and optical properties. ${ }^{1 a, b}$ When this interest seemed to be waning it received an unexpected

(1) (a) Gutman, F.; Lyons, L. E. Organic Semiconductors; Wiley: New York, 1967. (b) Meier, H. Organic Semiconductors; Verlag Chemie: Weinheim, 1974. boost from electropolymerization methods producing a conducting polymer which was "uniformly" doped, usually with a counteranion from the electrolyte used in the electropolymerization medium. ${ }^{2}$ The variety of polymers produced under an enormous range of operating conditions soon mushroomed, outgrowing the number

(2) Handbook of Conducting Polymers; Skotheim, T. A., Ed.; Marcel Dekker: New York, 1986; Vol. 1 and 2. 